\title{
State of the Art in Substance Use Prevention and Early Intervention: Applications to Pediatric Primary Care Settings
}

\author{
Pamela A. Matson ${ }^{1}$ (D) Ty Ridenour ${ }^{2} \cdot$ Nicholas lalongo $^{3} \cdot$ Richard Spoth $^{4} \cdot$ Guillermo Prado $^{5}$. \\ Christopher J. Hammond ${ }^{1}$. J. David Hawkins ${ }^{6} \cdot$ Hoover Adger Jr. $^{1}$
}

Accepted: 14 September 2021 / Published online: 29 October 2021

(c) Society for Prevention Research 2021

\begin{abstract}
With changes to drug-related policies and increased availability of many drugs, we currently face a public health crisis related to substance use and associated health consequences. Substance use and substance use disorders (SU/SUDs) are complex developmental disorders with etiologies that emerge through the intergenerational transmission of biological, familial, and environmental factors. The family ecosystem both influences and is influenced by SU/SUDs, particularly in children and adolescents. Family dynamics and parent functioning and behaviors can represent either risk or protective factors for the development of SU/SUDs in children. Primary care providers who provide care for children, adolescents, and families are in an ideal position to deliver prevention messages and to intervene early in the development of substance misuse and SUD among their patients. Despite recommendations from the American Academy of Pediatrics, few pediatric primary care providers provide anticipatory guidance to prevent or screen for substance misuse. Many barriers to those practices can be overcome through the integration and application of findings from the field of prevention science and the many lessons learned from the implementation of evidence-based interventions. Consideration of the implications of prevention science findings would help clarify the relevant roles and responsibilities of the primary care clinician, and the benefit of referral to and consultation from addiction specialists. Additionally, the past decade has seen the development and validation of a continuum of evidence-based prevention and early SU/SUD intervention activities that can be adapted for use in primary care settings making wide-spread implementation of prevention feasible. We propose a paradigm shift away from a model based on diagnosis and pathology to one upstream, that of family-focused prevention and early intervention. Adapting and scaling out empirically based prevention and early SU/SUD interventions to primary care settings and removing barriers to collaborative care across primary care, addiction medicine, and mental health providers offer the potential to meaningfully impact intergenerational transmission of SU/SUD — addressing a leading health problem facing our nation.
\end{abstract}

Pediatric health care professionals providing primary care for children and youth routinely encounter patients and family members who are at risk for or affected by alcohol,

Pamela A. Matson

pmatson@jhmi.edu

Johns Hopkins School of Medicine, Baltimore, MD, USA

2 RTI International, Research Triangle Park, Durham, NC, USA

3 Johns Hopkins Bloomberg School of Public Health, Baltimore, MD, USA

4 Iowa State University Partnerships in Prevention Science Institute, Ames, IA, USA

5 University of Miami School of Nursing and Health Studies, Miami, FL, USA

6 Social Development Research Group, University of Washington, Seattle, WA, USA tobacco, and other drug use, misuse, and related disorders. Addiction is a disease that frequently begins in adolescence or even childhood. We need to consider drug misuse as a developmental, pediatric disease. Pediatric primary care clinicians are in an ideal position to intervene in this growing, critically important health problem by providing prevention, early intervention, and/or referral to preventive and treatment services (Shah et al., 2016).

The importance of tobacco, alcohol, and other drug misuse as a pediatric health problem stems from its high prevalence among pediatric patients and their families, as well as the serious associated consequences. It is estimated that one in four children in the USA grows up in a household that is affected by alcohol abuse or dependence (Grant, 2000). Conducting health risk appraisal and preventive counseling throughout childhood, preadolescence, adolescence, and young adulthood is a well-established guiding principle in 
primary care. The need to incorporate into practice developmentally appropriate preventive interventions that strengthen families and maximize the health of children and adolescents is essential now more than ever in the era of legalized recreational marijuana for adults, the widespread prescription opioid use epidemic, and emerging data on COVID-19-related increases in levels of substance misuse (American Medical Association, 2021).

Despite longstanding recommendations from the American Academy of Pediatrics, few pediatric primary care providers screen for or provide anticipatory guidance to prevent substance use in their practice (Kuhns et al., 2020). Providers have been reluctant to answer this call to address substance misuse citing insufficient time, unfamiliarity with screening tools, feeling ill-equipped to handle a positive screen, not having knowledge of or access to appropriate referrals, worry about confidentiality, and the potential of offending parents (Van Hook et al., 2007). Many of these barriers can be overcome through the systematic application of findings from the decades of research in the field of prevention science, along with clarifying the roles and responsibilities of the primary care clinician.

\section{Call for a Paradigm Shift}

Pediatric primary care providers can play a vital role on the front line for prevention through addressing important factors related to delaying initiation and/or escalation of substance use. A paradigm shift is necessary to move from a practice model based on diagnosis and pathology to one upstream, with a range of prevention activities, particularly family-focused prevention (Leslie et al., 2016). Changes in health care and a growing toolbox of empirically validated prevention interventions that have to date only been implemented on a small scale in primary care provide emerging opportunities for family-focused and other prevention and early interventions administered in primary care (Koh et al., 2016).

\section{Application of Lessons Learned from Prevention Science to Primary Care Practices}

Prevention science has long recognized the need to address substance use early in childhood, by reducing risk factors and bolstering protective factors associated with substance use (Hawkins et al., 1992). A large body of evidence shows preventive interventions lower incidence of substance use and substance use disorders (SU/SUD) (Robertson et al., 2003). As family and school are the primary socializing environments for children, significant efforts have been expended to bring evidence-based familyfocused interventions to schools (Spoth et al., 2015). While ideal settings to reach children and parents, SU prevention is not the primary focus of the education mission. Few parents take advantage of these programs (Spoth \& Redmond, 2000). Parents do not see school-supported programs as legitimate sources for parenting advice, and fear of stigmatization discourages them from engaging in these prevention programs (Leslie et al., 2016). Pediatric providers are, however, in an ideal position to engage parents. Parents who have behavioral and mental health concerns for their children often seek care from pediatric providers (Thielke et al., 2007). Pediatric providers are highly trusted by parents, and parents often seek preventive care and anticipatory guidance from them (Leslie et al., 2016). Pediatric primary care settings are ideally suited to increase access of children, adolescents, and families to evidence-based SU prevention interventions. At least three core lessons from prevention science can be applied to pediatric primary care settings: (1) using a set of evidence-based universal prevention strategies, (2) clarity of messaging based on an accurate understanding of the science, and (3) engagement with families. Below outlines how a collaborative team-based approach with prevention scientists and clinical leaders working together to implement these lessons learned from the development and validation of a continuum of evidence-based prevention and early intervention activities that can be adapted for use in primary care settings making widespread implementation of prevention feasible and assisting primary care providers from becoming immobilized by the perceived increase in the burden of demands on their time.

\section{A Continuum of Prevention, Early Intervention, and Treatment Strategies}

Prevention, early intervention, and treatment strategies are employed depending on the patient's level of risk. Within each level of intervention, there is a continuum, or tiers, of involvement by the primary care provider, which range from minimal to significant investment of time and resources. Table 1 outlines this continuum of involvement within levels of universal, selective, and indicated prevention; early intervention and treatment strategies for primary care providers and within each of these levels; the possible roles of pediatric primary care providers in substance misuse prevention, and beginning with the least to the most demanding or resource intensive, Tier I to Tier III, respectively. 
Table 1 Type of prevention and continuum of prevention activities for pediatric primary care settings

Level I: Least demanding or resource intensive activities

Universal - Screening for substance misuse and substance use exposure

- Provide anticipatory guidance on substance misuse and substance use exposure

- Provide information (i.e., brochures/ handouts)

- Share with parents and adolescents the helpline and links to the information made available via Parents for Drug Free Kids and NIDA

- Referring parents to online parenting programs

Selective $\quad$ Monitor for needed prevention

- Make sub-specialty referral

Indicated $\bullet$ Make sub-specialty referral

- Refer for indicated prevention or treatment based on screening

Level II: Moderately demanding or resource intensive activities

- Designate an office champion to implement comprehensive screening

- Familiarize with talking tools and messaging (e.g., marijuana tool kit)

- Screen for liability of substance misuse or abuse before it occurs

- Providing an online parenting program and supporting parent engagement

- Refer for selective prevention based on screening

- Refer parent to recovery support programs

- Refer parent to treatment

- Conduct more formal evaluation/assessment

- Conduct motivational interviewing to promote behavior change

- Develop a management plan

- Make a subspecialty referral and follow-up on completing the referral
Level III: Most demanding or resource intensive activities

- Host on-site evidence based, family-focused education programs

- Link to community resources

\author{
.
}


screening instruments before the well-child visit. The Youth Risk Index ${ }^{\odot}$ is a valid screening instrument that generates individualized results, along with recommendations and materials; it has been shown to be feasible and acceptable with patients, physicians, and nurses (Ridenour et al., 2015). A negative screen provides the clinician with an opportunity to reinforce abstinence and to praise healthy choices, while a positive screen indicates a need for additional assessment.

Pediatric providers frequently have been hesitant to engage in screening feeling ill-equipped to handle a positive screen (Van Hook et al., 2007). While pediatric providers play a critical role in identifying substance misuse, they are not expected to manage or treat the problem in isolation. Just as the expertise of prevention scientists can be leveraged for prevention strategies, providers can consult with addiction specialists when they identify substance misuse through routine screening. Addiction medicine is a formally recognized medical subspecialty that brings the prevention of substance use and substance use disorders and the treatment and management of addiction into mainstream American medicine (O'Connor et al., 2014). Addiction specialists can support more formal assessment and evaluation of SU as well as assist with implementation of intervention and treatment plans.

Pediatric Practices as Hosts of Family-focused Interventions Primary care-based parenting interventions have been shown to impact positive parenting behaviors and substance use (Shah et al., 2016). Parent skills training, including parental monitoring, parental supportiveness, and parentchild communication, is vital for prevention and treatment of children's substance-related emotional and behavior problems (Breitenstein et al., 2014; NIDA, 2020). To increase parent engagement, pediatric primary care practices can provide online parenting programs (Tier II), which have been found to be feasible and impactful on both parent and child outcomes(Breitenstein et al., 2014). As an example, an online adaption of Familias Unidas, a prevention intervention currently being evaluated in primary care practices (Prado et al., 2019), has been shown to be efficacious at reducing SU/SUD (Estrada et al., 2019). Physicians link parents to the Familias Unidas program, which consists of online support modules as well as dynamic, engaging (i.e., Telenovela), and culturally tailored prevention messages (Estrada et al., 2017). Evidence-based family-focused preventive interventions can be delivered universally through the primary care provider's office by staff trained to deliver the program or delivered digitally through the internet. With a greater investment in time and resources (Tier III), pediatric primary care practices can serve as the hosts and supporters of more intensive, effective universal preventive interventions for all families. Applying a universal approach removes the stigma of patients and families being called out for being "at risk" and makes it clear that this is something all parents should do for their child's health. Pediatric primary care offers the potential to normalize attendance in parenting programs as is common with obstetrics clinics offering childbirth and infant care classes (Leslie et al., 2016). Pediatric primary care providers can provide clear and consistent recommendations to parents of all children entering adolescence to participate in a family-focused SU/SUD preventive intervention, whether offered through their clinic, online, or in the community. This can be combined with community-based campaigns promoting parents' expectations regarding participation in tested and effective parenting programs (Leslie et al., 2016; NIDA, 2020). Consistent recommendation and endorsement by the primary care provider to engage in prevention and early intervention will make a significant impact on program uptake; however, primary care providers may need to tailor programs to reflect the cultural or regional context of the patients and families in their practice. Further, understanding clinic workflow may be important to determine whether the intervention requires adaptations; for instance, in some cases, clinic workflow may allow part of an intervention to be delivered on site. Collaborative partnerships between researchers and primary care providers can be leveraged to maintain core components of evidence-based interventions while incorporating practice-initiated adaptations (Spoth et al., 2013).

Family History of Substance Use Pediatric providers typically are experts at communicating with parents respectfully to optimize the health of their patients. Assessing family history of substance misuse and SUD is a natural extension of this training. Multiple organizations recommend that screening for family history of SUD should be considered a core task of universal SU prevention in pediatric settings (Adger et al., 1999). Children with substance misusing parents are at greater risk for mental and physical health problems, maltreatment, and SUD (Walsh et al., 2003). Obtaining the family history of SU/SUD should be viewed through the same lens as obtaining a family history of other heritable health conditions (e.g., obesity, diabetes), with family history being obtained concurrent with family environmental factors (i.e., diet) with the goal of supporting optimal health for the child and preventing intergenerational transmission of disease. From the earliest engagement with a family, primary care providers have the opportunity to assess history of parental SU/SUD. Children of parents with SUD are genetically, socially, and environmentally pre-disposed to substance misuse and SUD, and as such it is necessary to engage parents early and repeatedly on SU history (Kumpfer, 1999). Providing substance-informed care increases our ability to prevent and/or minimize harms associated with addiction in the family. 
Screening, Brief Intervention, and Referral to Treatment (SBIRT) is an evidence-based practice used to identify, reduce, and prevent problematic use, misuse, and dependence on alcohol and other drugs (Substance Abuse Mental Health Service Administration, 2016). While increasingly adopted, the focus largely has been on identifying adolescents who are experiencing problems associated with use or addiction (Saitz et al., 2014). While SBIRT is an essential part of assessing the riskiness of SU behaviors and identifying problematic use and the need for treatment, the focus of screening should be broadened beyond the adolescent. SBIRT should be contextualized into a broader familyfocused approach (F-SBIRT) incorporating screening for SU/SUD in the family and household. This "wholistic" F-SBIRT approach has the potential to reduce adverse outcomes and prevent a child's SU exposure and/or development of SUD (Matson et al., 2021). While providers fear offending families, research shows that caregivers overwhelmingly endorse universal screening for SU/SUD in the family/household during well-child visits (Matson et al., 2021). Screening for parental SU provides a critical opportunity for selective or targeted prevention programs (Blueprints for Healthy Youth Development, 2015).

\section{Selective Prevention in Primary Care Settings}

Selective prevention programs are targeted to individuals with defined risk factors for the development of substance misuse and SUD, such as children who are assessed as having liability for SUD based on dispositional traits and/or coming from a family experiencing addiction (Ridenour et al., 2015). At a minimum, identifying that a patient is living in a home environment where parents and/or household members are misusing alcohol or other drugs or have untreated SUD allows the provider to view the health care needs of this child using a substance-informed lens. Studies indicate that children from families with SUD have greater health care needs overall, suggesting the need for closer monitoring of these patients and families (Ray et al., 2007). Primary care providers who identify a child as living in a home with concerning substance use can increase followup and monitoring of the child, demanding minimal practice resources (Tier I). With a moderate increase in time and investment, a provider can refer the child and family members to family-focused prevention programs, education, and recovery support (Tier II). The Family Checkup is one intervention option shown to be efficacious for the prevention of SU that providers could refer to that is family based and has an emphasis on motivational interviewing (Dishion et al., 2002). Getting a parent into care serves as a powerful preventive intervention for the child. Research has shown that individuals who successfully complete SUD treatment had medical cost reduction for spouses and children, pointing to improved health of the family as a whole (Weisner et al., 2010). Pediatric primary care providers are not expected to diagnose SUD or determine type or level of care for the parent. It is hopeful that with moderate additional involvement, providers will provide resources for families who express interest in treatment (Tier II). An additional strategy is to integrate mental health into the existing health care model (Briggs et al., 2007). One approach that has been shown to improve care coordination is to have a social worker or behavioral health specialist embedded in the practice, allowing for more enhanced, office-based, selective prevention and early intervention programs, and improved targeting of co-occurring mental health conditions (Tier III). While more resource intensive, with the integration of an ancillary service provider, the practice's ability to engage in all levels of preventive intervention from universal to indicated can be enhanced without an increased time burden on the primary care provider. Recent work has found that pediatricians overwhelmingly support screening and referral to family-based prevention and that co-locating family therapists (or behavioral specialists on their staff) into well-child visits could overcome several of the most prevalent barriers (lack of time, workflow, and concerns with compliance and parental support) (Ridenour et al., In process).

While selective interventions often are relatively more intensive for participants when compared with universal interventions, they do not necessarily require increasing demands on the provider. PROSPER and Communities that Care (CTC) partnership models provide a well-established prevention infrastructure that support evidence-based universal, selective, and indicated family-based prevention interventions in pediatric primary care settings. Pediatric primary care practices can engage with the PROSPER partnership model in their state (http://helpingkidsprosper. org) to access a menu of evidence-based programs (Spoth et al., 2015; Spoth et al., 2017). CTC employs a social development strategy to provide community-specific, prosocial opportunities for youth (www.communitiesthatcare. net) and has similarly shown reductions in rates of youth SU (Oesterle et al., 2018). Both models could be readily extended to link primary care practices to community prevention resources.

\section{Indicated Prevention in Primary Care Settings}

Indicated, or secondary, prevention strategies are aimed at individuals who are experiencing early signs of substance misuse. Indicated strategies aim to halt or slow progression of SU and to prevent development of SUD. Screening for any SU rather than for only problematic SU or SUD identifies adolescents who would benefit from indicated prevention strategies. A positive SU screen at any level of SU should be considered an exposure as opposed to viewing early or low 
level SU as experimentation ("normal kid stuff"). At the low end of the continuum of care, pediatric providers can make a sub-specialty referral for support from an Addiction Specialist or refer for indicated prevention or treatment based on screening results (Tier I).

With increased time and investment, pediatric providers can conduct more formal assessments. Primary care providers who want to treat patients who screen positive also could employ motivational interviewing techniques to foster behavior change, develop a management plan, and increase monitoring with short-term follow-up visits (Tier II). Providers who have a strong interest in substance misuse could participate in continuing education to learn how to manage patients at a higher level of complexity including the use of medications for SUD (Tier III).

\section{Overcoming Barriers to Family-Focused Prevention in Pediatric Primary Care}

To fundamentally address barriers to screening for substance misuse in primary care, there is an immediate need for action in medical training. Pediatric residency programs must include skills training and experiences to develop a level of comfort around screening, early intervention, and consultation with addiction medicine specialists. (O'Connor et al., 2011) Training should include developing competencies in early childhood screening and prevention programs, such as the Family Check-Up. (Dishion et al., 2002) Moreover, substance use-related education and training should hold a prominent place within the medical school curriculum. Increasing competence in screening and referral may overcome the perceived barrier that there is insufficient time to address substance use in pediatric primary care settings. Additional strategies to address time constraints would be to optimize screening into the workflow of primary care settings, specifically to incorporate standardized screening instruments into the electronic medical record system, which may be more impactful than provider factors (Sterling et al., 2012; Tai \& McLellan, 2012). Evidence supports the efficacy of using a computer- facilitated screening and brief advice system to increase substance use counseling by the primary care provider and to reduce adolescent substance use (Harris et al., 2012; Ridenour et al., 2015). Ultimately, to ensure sustainability of screening and prevention in primary care settings, reliable funding and reimbursement models must be addressed. The Affordable Care Act mandates public and private coverage of substance use screening and brief counseling during primary care visits and offers the potential for the integration of behavioral and physical health care, including an integrated structure for collaboration among physicians, nurses, social workers, and allied health professionals to provide screening, prevention, early intervention, and treatment (Hawkins et al., 2015). Innovative strategies for sustainable funding of evidence-based preventive interventions at community, county, and state levels including alternative payment models for children that build upon the Center for Medicare and Medicaid Innovation's Integrated Care for Kids Model (InCK) and shift incentives toward long-term health promotion and incentivize whole family health promotion have been proposed (Counts et al., 2021). Nonetheless, evaluation of adoption, implementation, and sustainability strategies provide important opportunities to facilitate provider decision making, organizational readiness, participant engagement, intervention fidelity and adaptation, financing strategies, and long-term maintenance of interventions in primary care (Spoth et al., 2013).

Knowing the prevalence of SUD among adults, many of whom are parents, and the knowledge that children of parents with SUD are at greater risk for SUD themselves suggests that we have a great number of youth in the pipeline headed for misuse of alcohol and other drugs. Primary care clinicians who see children, adolescents, and families are in an ideal position to deliver prevention messages and to intervene early in the development of substance misuse. Taking a "two-generation approach" recognizes that promoting health of the parent is health promotion for the child and family (Mosle \& Patel, 2012). Importantly, pediatric primary care providers are capable of prevention in some form and they have the support of both addiction specialists and prevention scientists. By our view, the time has come for clinicians to join forces with other child-care experts. These collective efforts offer the potential for a meaningful impact on addressing a leading health problem facing our nation.

Funding All phases of this study were supported in part by the National Institute of Drug Abuse (NIDA K01DA035387; PI Matson).

\section{Declarations}

Ethics Approval This article does not include human subjects.

Informed Consent This article does not include human subjects.

Conflict of Interest The authors declare no competing interests.

\section{References}

Adger, H., Macdonald, D. I., \& Wenger, S. (1999). Core competencies for involvement of health care providers in the care of children and adolescents in families affected by substance abuse. Pediatrics, 103, 1083-1084.

American Board of Addiction Medicine Foundation. (2013). Compendium of Educational Objectives for Addiction Medicine Residency Training.

American Medical Association. (2021). Issue brief: Reports of increases in opioid- and other drug-related overdose and other concerns during 
COVID pandemic. Retrieved from: https://www.ama-assn.org/system/ files/2020-11/issue-brief-increases-in-opioid-relatedoverdose.pdf

Blueprints for Healthy Youth Development. (2015). www. blueprintsprograms.com/allPrograms.php

Breitenstein, S. M., Gross, D., \& Christophersen, R. (2014). Digital delivery methods of parenting training interventions: a systematic review. Worldviews on Evidence-Based Nursing, 11, 168-176.

Briggs, R. D., Racine, A. D., \& Chinitz, S. (2007). Preventive pediatric mental health care: A co-location model. Infant Mental Health Journal, 28, 481-495.

Committee on Substance Abuse, Levy, S. J., \& Kokotailo, P. K. (2011). Substance use screening, brief intervention, and referral to treatment for pediatricians. Pediatrics, 128, 1330. https://doi.org/10. 1542/peds.2011-1754

Counts, N. Z., Roiland, R. A., \& Halfon, N. (2021). Proposing the ideal alternative payment model for children. JAMA Pediatrics.

Dishion, T. J., Kavanagh, K., Schneiger, A., Nelson, S., \& Kaufman, N. K. (2002). Preventing early adolescent substance use: a family-centered strategy for the public middle school. Prevention science : the official journal of the Society for Prevention Research, 3, 191-201.

Estrada, Y., Lee, T. K., Wagstaff, R., Rojas, L. M., Tapia, M. I., Velázquez, M. R., et al. (2019). eHealth Familias Unidas: Efficacy trial of an evidence-based intervention adapted for use on the internet with Hispanic families. Prevention Science, 20, 68-77.

Estrada, Y., Molleda, L., Murray, A., Drumhiller, K., Tapia, M., Sardinas, K., et al. (2017). eHealth Familias Unidas: pilot study of an internet adaptation of an evidence-based family intervention to reduce drug use and sexual risk behaviors among Hispanic adolescents. International journal of environmental research and public health, 14, 264.

Geoffrey, R. S., Cynthia, B., Graham, A. B., Brown, O. W., Hardin, A., Lessin, H. R., et al. (2014). Recommendations for pediatric preventive health care. Pediatrics, 133, 568-570. https://doi.org/ 10.1542/peds.2013-4096

Grant, B. F. (2000). Estimates of US children exposed to alcohol abuse and dependence in the family. American Journal of Public Health, 90, 112-115.

Harris, S. K., Csémy, L., Sherritt, L., Starostova, O., Van Hook, S., Johnson, J., et al. (2012). Computer-facilitated substance use screening and brief advice for teens in primary care: an international trial. Pediatrics, 129, 1072-1082.

Hawkins, J. D., Catalano, R. F., \& Miller, J. Y. (1992). Risk and protective factors for alcohol and other drug problems in adolescence and early adulthood: implications for substance abuse prevention. Psychological Bulletin, 112, 64.

Hawkins, J. D., Jenson, J. M., Catalano, R., Fraser, M. W., Botvin, G. J., Shapiro, V., et al. (2015). Unleashing the power of prevention. Discussion Paper, Institute of Medicine and National Research Council, Washington, DC.

Johnston, L. D., O’Malley, P. M., Miech, R. A., Bachman, J. G., \& Schulenberg, J. E. (2016). Monitoring the future national survey results on drug use, 1975-2015: Overview, key findings on adolescent drug use.

Koh, H. K., Rajkumar, R., \& McDonough, J. E. (2016). Reframing prevention in the era of health reform. JAMA, 316, 10391040. https://doi.org/10.1001/jama.2016.10405

Kuhns, L. M., Carlino, B., Greeley, K., Muldoon, A. L., Karnik, N., Thompson, H., et al. (2020). A chart review of substance use screening and related documentation among adolescents in outpatient pediatric clinics: implications for practice. Substance Abuse Treatment, Prevention, and Policy, 15, 1-6.

Kumpfer, K. L. (1999). Outcome measures of interventions in the study of children of substance-abusing parents. Pediatrics, 103, $1128-1144$

Leslie, L. K., Mehus, C. J., Hawkins, J. D., Boat, T., McCabe, M. A., Barkin, S., et al. (2016). Primary health care: Potential home for family-focused preventive interventions. American Journal of Preventive Medicine, 51, S106-S118. https://doi.org/10.1016/j. amepre.2016.05.014

Matson, P. A., Bakhai, N., Solomon, B. S., Flessa, S., Ramos, J., Hammond, C. J., Adger, H. (2021). Understanding caregiver acceptance of screening for family substance use in pediatric clinics serving economically disadvantaged children. Substance Abuse. Published online: 02 Jul 2021. https://doi.org/10.1080/ 08897077.2021.1941510 PMID: 34214411.

Mosle, A., \& Patel, N. (2012). Two generations, one future: Moving parents and children beyond poverty together. The Aspen Institute. Retrieved from ascend.aspeninstitute.org/resources/ two-generations-one-future

NIDA. (2016). Principles of substance abuse prevention for early childhood.

NIDA. (2020). Preventing drug use among children and adolescents (In Brief). https://www.drugabuse.gov/publications/preventingdrug-use-among-children-adolescents/introduction.

O’Connor, P. G., Nyquist, J. G., \& McLellan, A. T. (2011). Integrating Addiction Medicine Into Graduate Medical Education in Primary Care: The Time Has Come. Annals of Internal Medicine, 154, 56-59.

O'Connor, P. G., Sokol, R. J., \& D'Onofrio, G. (2014). Addiction medicine: The birth of a new discipline. JAMA internal medicine, 174, 1717-1718. https://doi.org/10.1001/jamainternmed.2014.4211

Oesterle, S., Kuklinski, M. R., Hawkins, J. D., Skinner, M. L., Guttmannova, K., \& Rhew, I. C. (2018). Long-term effects of the communities that care trial on substance use, antisocial behavior, and violence through age 21 years. American Journal of Public Health, 108, 659-665.

Prado, G., Estrada, Y., Rojas, L. M., Bahamon, M., Pantin, H., Nagarsheth, M., et al. (2019). Rationale and design for eHealth Familias Unidas Primary Care: A drug use, sexual risk behavior, and STI preventive intervention for hispanic youth in pediatric primary care clinics. Contemporary clinical trials, 76, 64-71.

Ray, G. T., Mertens, J. R., \& Weisner, C. (2007). The excess medical cost and health problems of family members of persons diagnosed with alcohol or drug problems. Medical Care, 45, 116-122. https://doi. org/10.1097/01.mlr.0000241109.55054.04

Ridenour, T. A., Desiree W. Murray, D. W., Hinde, J., Cristie, G., Wilkinson, A., Rackers, H., et al. (In process). Addressing barriers to primary care screening and referral to prevention for youth risky health behaviors: Evidence regarding potential cost-savings and provider concerns. Prevention Science.

Ridenour, T. A., Willis, D., Bogen, D. L., Novak, S., Scherer, J., Reynolds, M. D., et al. (2015). Detecting initiation or risk for initiation of substance use before high school during pediatric well-child check-ups. Drug and Alcohol Dependence, 150, 54-62. https://doi.org/10.1016/j. drugalcdep.2015.02.013

Robertson, E. B., David, S. L., \& Rao, S. A. (2003). Preventing drug use among children and adolescents: A research-based guide for parents, educators, and community leaders (Vol. Book, Whole): Diane Publishing.

Saitz, R., Palfai, T. P., Cheng, D. M., Alford, D. P., Bernstein, J. A., Lloyd-Travaglini, C. A., et al. (2014). Screening and brief intervention for drug use in primary care: The ASPIRE randomized clinical trial. JAMA, 312(5), 502-513. https://doi.org/10.1001/ jama.2014.7862

Shah, R., Kennedy, S., Clark, M. D., Bauer, S. C., \& Schwartz, A. (2016). Primary care-based interventions to promote positive parenting behaviors: A meta-analysis. Pediatrics, 137, e20153393.

Spoth, R., \& Redmond, C. (2000). Research on family engagement in preventive interventions: Toward improved use of scientific findings in primary prevention practice. Journal of Primary Prevention, 21, 267-284.

Spoth, R., Redmond, C., Mason, W., Schainker, L., \& Borduin, L. (2015). Research on the Strengthening Families Program for 
parents and youth 10-14: Long-term effects, mechanisms, translation to public health, PROSPER partnership scale up.

Spoth, R., Redmond, C., Shin, C., Greenberg, M., Feinberg, M., \& Trudeau, L. (2017). PROSPER delivery of universal preventive interventions with young adolescents: long-term effects on emerging adult substance misuse and associated risk behaviors. Psychological Medicine, 47, 2246.

Spoth, R., Rohrbach, L. A., Greenberg, M., Leaf, P., Brown, C. H., Fagan, A., et al. (2013). Addressing core challenges for the next generation of type 2 translation research and systems: The translation science to population impact (TSci Impact) framework. Prevention Science, 14, 319-351.

Sterling, S., Kline-Simon, A. H., Wibbelsman, C., Wong, A., \& Weisner, C. (2012). Screening for adolescent alcohol and drug use in pediatric health-care settings: Predictors and implications for practice and policy. Addiction science \& clinical practice, 7, 1-12.

Substance Abuse Mental Health Service Administration. (2021). Screening, brief intervention, and referral to treatment (SBIRT). (Vol. 2016). Retrieved from: http://www.samhsa.gov/sbirt

Tai, B., \& McLellan, A. T. (2012). Integrating information on substance use disorders into electronic health record systems. Journal of substance abuse treatment, 43, 12-19.
Thielke, S., Vannoy, S., \& Unützer, J. (2007). Integrating mental health and primary care. Primary Care: Clinics in Office Practice, 34, 571-592. https://doi.org/10.1016/j.pop.2007.05.007

Van Hook, S., Harris, S. K., Brooks, T., Carey, P., Kossack, R., Kulig, J., et al. (2007). The "Six T's": Barriers to screening teens for substance abuse in primary care. J Adolesc Health, 40, 456-461. https://doi.org/ 10.1016/j.jadohealth.2006.12.007

Volkow, N. D., Baler, R. D., Compton, W. M., \& Weiss, S. R. (2014). Adverse health effects of marijuana use. The New England Journal of Medicine, 370, 2219-2227. https://doi.org/10.1056/NEJMra1402309

Walsh, C., MacMillan, H. L., \& Jamieson, E. (2003). The relationship between parental substance abuse and child maltreatment: Findings from the Ontario Health Supplement. Child Abuse \& Neglect, 27, 1409-1425. S0145213403002400[pii]

Weisner, C., Parthasarathy, S., Moore, C., \& Mertens, J. R. (2010). Individuals receiving addiction treatment: are medical costs of their family members reduced? Addiction (Abingdon, England), 105, 1226-1234. https://doi.org/10.1111/j.1360-0443.2010. 02947.x

Publisher's Note Springer Nature remains neutral with regard to jurisdictional claims in published maps and institutional affiliations. 